\title{
Evolution of cluster production with fragmentation degree
}

\author{
E. BONNET \\ SUBATECH UMR 6457, IMT Atlantique, Université de Nantes, CNRS-IN2P3 \\ 44300, Nantes, France
}

received 3 December 2018

\begin{abstract}
Summary. - We present in this work, the experimental production of clusters in the ${ }^{58} \mathrm{Ni}+{ }^{58} \mathrm{Ni}$ reactions at incident beam energies $\left(E_{\text {inc }}\right)$ between 32 and $90 \mathrm{MeV} / A$ collected with the INDRA apparatus. We focus on the evolution of their contribution respect to the final fragmentation degree given by the charge of the biggest fragment $\left(Z_{1}\right)$ of each event. Mean multiplicities increase when $Z_{1}$ decreases, but show a plateau for intermediate $Z_{1}$ values, before increasing again toward complete vaporization. This break is in coincidence with the multifragmentation window and is more clear at $32 \mathrm{MeV} / A$. The opening of the fragment production has an energy cost making the production of clusters in the same time impossible. Using the mass fraction observable $\left(X_{A}\right)$, we look at probabilities of nucleons to be free or bound in clusters and how the different species evolve with fragmentation degree and with the incident energy of the collision. We highlight the specific role of the ${ }^{4}$ He clusters in the whole range of fragmentation degree. We show that their evolution shape can be used to sign the multifragmentation window. Looking at the different incident energies, The main effect of increasing the average deposited energy in the system is an increase of final light clusters $\left({ }^{2,3} \mathrm{H},{ }^{3} \mathrm{He}\right)$ at the expence of the heavier ones.
\end{abstract}

\section{1. - Motivations}

Understanding the production of clusters (namely nucleons ended together) in heavyion collisions (HIC) is a hot and hard topic. Bringing experimentally constraints on that topic should help to constrain both EOS of non-homogeneous nuclear matter (NM) and transport models. Experimentally, having access to the properties of the region where clusters emerge and extracting the time scale of their production respect to the time scale of the whole collision is a great challenge. As a first step, we will study contributions of clusters to final partitions regardless of the underlying mechanisms that produced these clusters. We just use a sorting observable linked with the fragmentation degree of the partitions. We do that because, depending on the fragmentation degree associated to different excitation/dissipation/violence, the available nucleons to build "clusters" is not 
the same. While for evaporation events, final partitions are made of heavy residues surrounded by free nucleons and clusters, for vaporization events, all nucleons are available to contribute to the cluster production. Inside final partitions, we choose the charge of the biggest fragment $\left(Z_{1}\right)$ as a straightforward and robust estimation of the fragmentation degree. Using it, we can draw a continuous evolution between evaporation (high $Z_{1}$ values, low fragmentation degree) and vaporization (low $Z_{1}$ values, high fragmentation degree) passing through the multifragmentation process (intermediate values of $Z_{1}$ and fragmentation degree).

In this general picture, we will address the contribution of the different species to the final partitions produced in the ${ }^{58} \mathrm{Ni}+{ }^{58} \mathrm{Ni}$ reactions. First we will introduce briefly the experimental data and the selection prior to the analysis. Then, we will present mean evolution of multiplicities and mass fractions according to $Z_{1}$. Finally, we will discuss the influence of the deposited energy looking at three different beam energies $\left(E_{\text {inc }}\right)$.

\section{2. $-{ }^{58} \mathrm{Ni}+{ }^{58} \mathrm{Ni}$ reactions}

The ${ }^{58} \mathrm{Ni}+{ }^{58} \mathrm{Ni}$ reactions, collected with the INDRA apparatus, have been already studied to probe the isospin diffusion in semi-peripheral collisions [1] and to extract fusion cross sections in light systems with a significant contribution at $E_{i n c}=32$ and $40 \mathrm{MeV} / A$ [2]. Detailed description of the experimental set-up and the data taking can be found in the related publications. Data acquisition was triggered by events with at least 4 particles. From this set of collected events, we only use the forward part of each event corresponding to charged products with velocity greater than the centre-of-mass velocity. These products constitute the partitions under study. To maximize information and get reliable results, only events with a missing charge $\left(Z_{\text {missing }}\right)$ less or equal to 4 compared to the ${ }^{58} \mathrm{Ni}$ charge are kept: $Z_{\text {missing }}=\left(28-\sum_{j=1}^{m_{\text {tot }}} Z_{j}\right) \leq 4$. The percentage of selected events varies between $40 \%$ and $25 \%$ respectively for $E_{\text {inc }}=32 \mathrm{MeV} / A$ and $90 \mathrm{MeV} / A$. From that selection, we are sure that the heaviest fragment $\left(Z_{1}\right)$ is part of the partition and therefore, we are confident about the quality of the fragmentation degree sorting used in the analysis.

\section{3. - Results}

3·1. Multiplicities. - On the first panel of fig. 1, the $Z_{1}$ distributions are shown for the selected events and for the three beam energies used in the analysis. The whole range of values are populated and the proportion of vaporization events increases with bombarding energies at the expense of evaporation events. The use of a rather small system, ${ }^{58} \mathrm{Ni}+{ }^{58} \mathrm{Ni}$, makes the detection of complete events on the whole range of fragmentation degree easier, especially focusing only on the forward part.

The other panels of fig. 1 show different multiplicities evolution with $Z_{1}$. From detailed analysis, not shown in this paper, we have identified different groups which exhibit the same trend. We introduce here the different groups which will be used in the following: as a reference, "All" stands for all detected charged products, " $A=1$ ", stands for protons, " $A=2 \& 3$ " stands for ${ }^{2,3} \mathrm{H}$ and ${ }^{3} \mathrm{He}$ isotopes, " $A=4$ " stands for ${ }^{4} \mathrm{He}$ isotopes and " $A>4$ " corresponds to the heavier charged products starting with ${ }^{6} \mathrm{He}$. First we focus on the lowest $E_{i n c}, 32 \mathrm{MeV} / A$ (open circles): we observe as a general trend, an increase of the multiplicities when $Z_{1}$ is decreasing. This trivial behaviour is only due to the conservation of the number of nucleons when a sorting using $Z_{1}$ is done. The interesting point is that, already for $m_{A l l}$, we observe an inflexion point in this evolution starting 

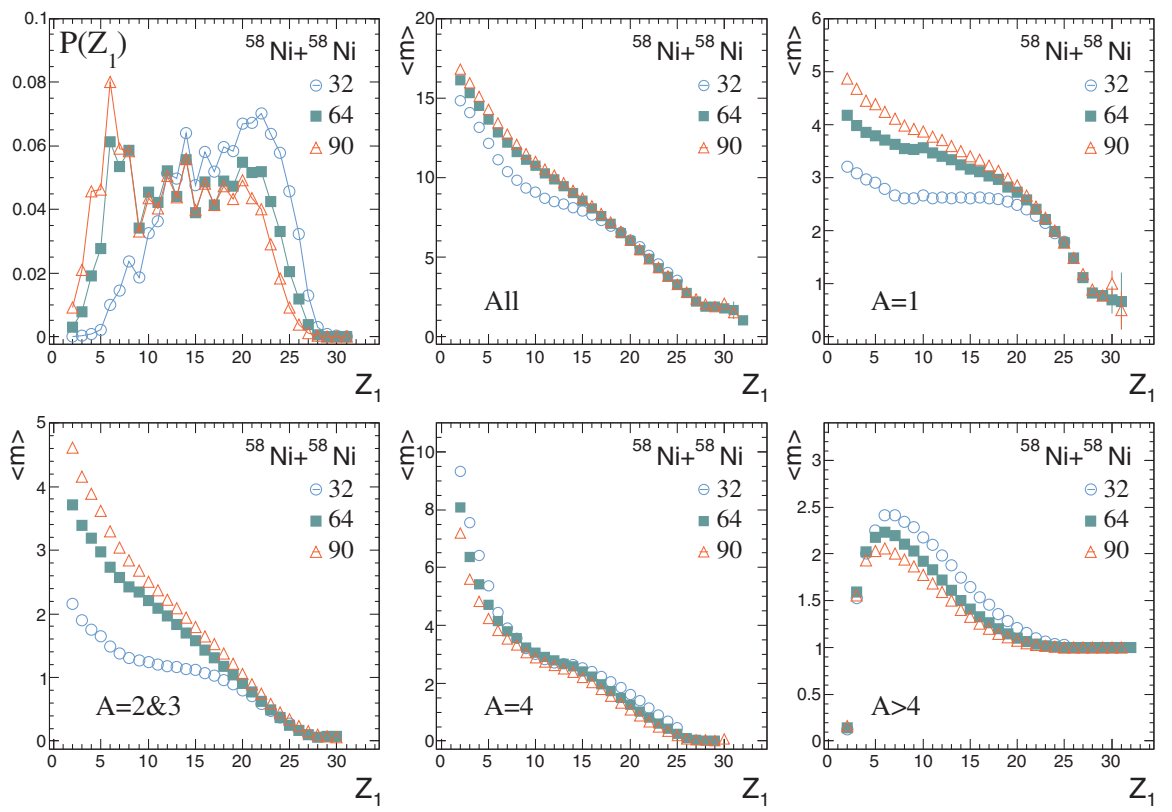

Fig. 1. - Results for ${ }^{58} \mathrm{Ni}+{ }^{58} \mathrm{Ni}$ reactions for the three incident beam energies: open circles for $32 \mathrm{MeV} / A$, full squares for $64 \mathrm{MeV} / A$ and open triangles for $90 \mathrm{MeV} / A$. Top, from left to right: normalized $Z_{1}$ distributions, mean evolution with $Z_{1}$ of the multiplicity of all charged products, mean evolution with $Z_{1}$ of the multiplicity of protons $(A=1)$. Bottom, from left to right, mean evolution with $Z_{1}$ of the multiplicity of: ${ }^{2,3} \mathrm{H}$ and ${ }^{3} \mathrm{He}$ isotopes $(A=2 \& 3),{ }^{4} \mathrm{He}$ isotopes $(A=4)$ and heavier charged products $(A>4)$.

around $Z_{1}=15$. In coincidence to this inflexion point, we observe a plateau for $m_{A=1}$, $m_{A=2 \& 3}$ and $m_{A=4}$ followed by an increase for $Z_{1} \sim 6-8$. The localization of this plateau corresponds roughly to the region where the production of the heavier charge product $(A>4)$ is significant and reach its maximum. The observed sequence in the cluster production when increasing the fragmentation degree can be described as follows: an evaporation regim associated to cluster production, a break in this production associated to heavier products when multifragmentation starts to act and finally a vaporization regim where the system is composed of free nucleons and light clusters. The production break is an echo of the energy cost to produce fragment surfaces as expected in this multifragmentation regim.

If now we look at higher beam incident energies $(64 \mathrm{MeV} / A$, full squares and $90 \mathrm{MeV} / A$, open triangles), the effects are the following. For low fragmentation degree $\left(Z_{1}>20\right)$, the different $E_{i n c}$ give almost the same results. For lower $Z_{1}$ values, the inflexion point of $m_{A l l}$ is progressivly washed out. Plateaux observed for $m_{A=1}$ and $m_{A=2 \& 3}$ are also filled and the trends become monotonic while for ${ }^{4} \mathrm{He}$, the plateau remains. For a given $Z_{1}$ value, $m_{A=1}$ and $m_{A=2 \& 3}$ values increase with $E_{i n c}$, while for $m_{A=4}$ and $m_{>4}$, values decrease. We pointed out, here a migration of nucleons from " $A=4$ " and " $A>4$ " groups to " $A=1$ " and " $A=2 \& 3$ " groups.

For $E_{i n c}=32 \mathrm{MeV} / A$, the sequence: evaporation, multifragmentation and vaporization is deduced from the presence of a plateau in the different multiplicities evolution. It fits the picture of the successive opening of processes close to their energy threshold leading to production of "cold partitions". For higher $E_{i n c}$, an additional contribution 
in the production of clusters are signed with a general shift of multiplicities on the whole range of $Z_{1}$. This is coherent with a second step process contribution from excited partitions which blurs the previous sequence.

To go further in the analysis we have to take into account the trivial trends observed such as the general increase of $m_{A l l}$ with fragmentation degree but also the fact that for one $Z_{1}$ values to another, the number of nucleons in the remaining part is not the same.

3.2. Mass fractions. - We now look at the evolution of the cluster contributions in the remaining part. To do so, we introduce for each species the mass fraction as written in eq. (1)

$$
X_{A}^{(i)}=m_{i} A_{i} /\left(\sum_{j=1}^{m_{t o t}} A_{j}-A_{1}\right)
$$

The number of nucleons bound in the $Z_{1}$ fragment are removed in the normalization. Then, the mass fraction is the probability for a nucleon to belong to one or the other of the species in the remaining part. This allows us to get rid of the different number of nucleons available when the fragmentation degree evolves. In fig. 2, mean evolutions of the mass fraction are shown for the groups previously defined. The three panels correspond to the following three incident beam energies: 32,64 and $90 \mathrm{MeV} / A$.

The first difference using mass fraction appears looking at protons. They present a continuous decrease ending with a saturation below $Z_{1}=15$ values and this for all $E_{\text {inc }}$. For " $A=2 \& 3$ " and " $A=4$ " groups, plateaux previously observed for the multiplicities turn into rollercoaster shapes. The two branches are an echo of the pure evaporation and vaporization regims while the localization of their minimums around $Z_{1}=8-10$ is in a clearer coincidence with the maximum production of " $A>4$ " group. Concerning the nucleon probability, except for very low fragmentation degree where protons are predominant, the probability of the nucleons to be bound in ${ }^{4} \mathrm{He}$ isotopes dominate on the whole range of fragmentation degree. The nucleon migration between " $A=4$ " and " $A>4$ " groups to " $A=1$ " and " $A=2 \& 3$ " remains in this representation and the influence of deposited energy and its possible link with time scale of fragment and cluster production will be investigated in further analysis.

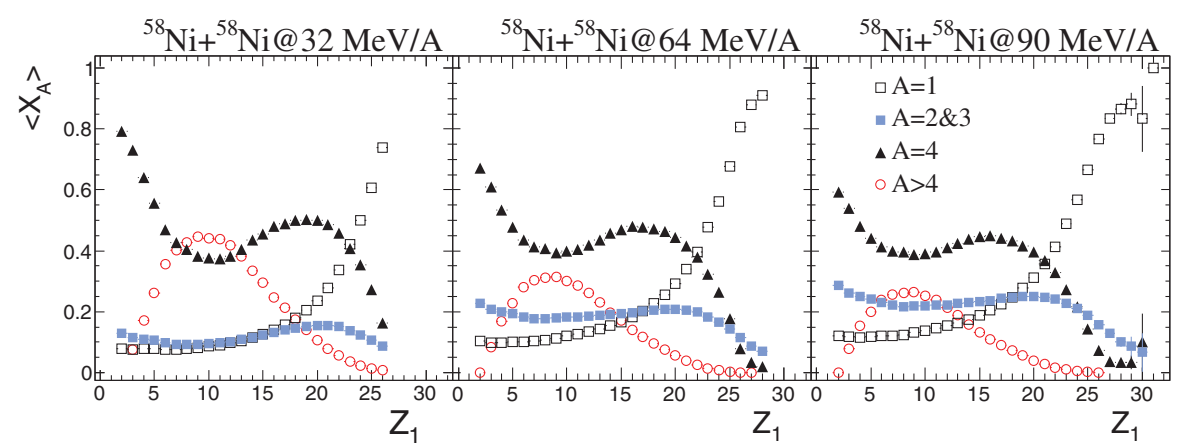

Fig. 2. - Results for ${ }^{58} \mathrm{Ni}+{ }^{58} \mathrm{Ni}$ reactions for incident beam energies 32,64 and $90 \mathrm{MeV} / A$ (from left to right). Evolution of the mass fraction $\left(X_{A}\right)$ for the different groups with the charge of the biggest fragment $\left(Z_{1}\right)$. Each symbol stands for: open squares for protons $(A=1)$, full squares for ${ }^{2,3} \mathrm{H}$ and ${ }^{3} \mathrm{He}$ isotopes $(A=2 \& 3)$, full triangles for ${ }^{4} \mathrm{He}$ isotopes $(A=4)$ and open crosses for heavier charged products $(A>4)$. 


\section{4. - Conclusion and outlook}

We have looked at the contribution of the different species which composed final partitions produced in the ${ }^{58} \mathrm{Ni}+{ }^{58} \mathrm{Ni}$ reactions. Using $Z_{1}$ as sorting observable, we have sampled classes of events according to their fragmentation degree, exploring successively evaporation, multifragmentation and vaporization. At $E_{i n c}=32 \mathrm{MeV} / A$, the presence of a plateau in the different multiplicity evolutions indicates the competition between production of clusters and heavier fragments due to the energy conservation while for higher $E_{\text {inc }}$, the clusters production are facilitated by the more important deposited energy in the systems. Looking at mass fraction: the $A>4$ contribution are clearly framed by the light clusters contribution for all $E_{\text {inc }}$ and the ${ }^{4} \mathrm{He}$ contribution is predominant whatever the underlying mechanism leading to final observed partitions.

The present results are averaged over a deposited energy distribution. We plan to go further in the analysis using an additional excitation energy $\left(E^{*}\right)$ sorting to see if the general picture is the same.

\section{REFERENCES}

[1] Galichet E. et al., Phys. Rev. C, 79 (2009) 064614.

[2] Lautesse Ph. et al., Eur. Phys. J. A, 27 (2006) 349. 